\title{
Morphological Neural Networks with Dendrite Computation: A Geometrical Approach
}

\author{
Ricardo Barrón, Humberto Sossa, and Héctor Cortés \\ Centro de Investigación en Computación - IPN \\ Av. Juan de Dios Bátiz S/N Esq. Miguel Othón de Mendizábal \\ Unidad Profesional "Adolfo López Mateos", Zacatenco \\ Mexico. D.F. C.P. 07738, MEXICO \\ Tel. 57296000 ext. 56560 \\ \{rbarron, hsossa\}@cic.ipn.mx, torinja@yahoo.com.mx
}

\begin{abstract}
Morphological neural networks consider that the information entering a neuron is affected additively by a conductivity factor called synaptic weight. They also suppose that the input channels account with a saturation level mathematically modeled by a MAX or MIN operator. This, from a physiological point of view, appears closer to reality than the classical neural model, where the synaptic weight interacts with the input signal by means of a product; the input channel forms an average of the input signals. In this work we introduce some geometrical aspects of dendrite processing that easily allow visualizing the classification regions, providing also an intuitive perspective of the production and training of the net.
\end{abstract}

\section{Introduction}

Neural networks are today a computational alternative to solve problems where is difficult or does not exist an algorithmic solution. Inspired on the functioning of the nervous system, researchers have postulated different neural processing models.

Recently, it has been found that information processing occurs also at dendrite level and not only at the neuron body [4]. This could be an explanation of the efficiency of the nervous system; due to the information processing practically occurs on the communication channel. This with morphological paradigm is starting point of this research.

\subsection{Outline of the Paper}

The remainder of the paper is organizes as follows. In Section 2, we briefly talk about the related work with the present research. In Section 3, we describe the adopted methodology to give a solution to the problem. In Section 4, we provide an example to explain the functioning of the proposed methodology. Finally, in Section 5, we conclude and give directions for future research. 


\section{State of the Art}

A neural network can be conceptualized as a non-linear mapping between two pattern-spaces: the input pattern and the output pattern. Normally the internal parameters of this mapping are determined by a training procedure and they are called, in most cases, synaptic weights.

In the decade of the 50's Rosenblatt [1] introduces the Perceptron. This classical model has served a basis of most of the actual developments.

\section{The Adopted Methodology}

\subsection{Morphological Neural Processing}

Morphological processing is based on the lattice algebra: $(R, \wedge,+)$, where $\wedge$ is the MIN operator [2]. The main property of this algebraic structure is distributivity of summation with respect to operator $\wedge$, this is:

$$
a+(b \wedge c)=(a+b) \wedge(a+c)
$$

From the point of view of neural processing, Ritter [4] proposes a model of neuron where the synaptic weights interact additively with the input signals; the dendrites discriminate by taking into account the minimal value of the incident signals, (see Figure 1).

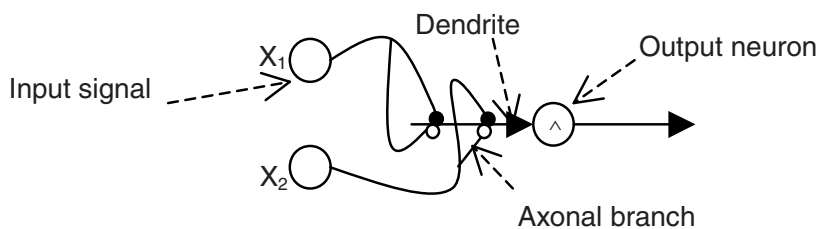

Fig. 1. Model of a morphological neuron.

In this model each branch can be of excitation or inhibition (excitation branches end with a black circle). The output neuron might have several dendrites; the output of each one of them can be negated or not.

A fundamental difference with respect to the neuronal classical model is that in morphological processing discrimination among input signals is done by taking into account a threshold that depends on a min value. In the classical model a weighted average of the inputs is taken. Since the physiological point of view it appears more acceptable the threshold criteria, although the quality of the models, it what we want to emulate is a biological process, only could be judged through the insights and scientific experiments in the area. 


\subsection{Dendrite Computation on Morphological Neurons}

One of the contributions of the model described in Section 3.1 is the capacity to accomplish processing practically over the same communication channel. In this case the axonal branches can be of excitation or inhibition; only at the moment of contact with the dendrite, just the MIN of the values remains, this is

$$
\bigwedge_{l \in L}(-1)^{1-l}\left(x_{i}+\omega_{i k}^{l}\right), \quad L=\{0,1\}
$$

is the value that filters dendrite $k$, where $x_{i}$ is the input and $\omega_{i k}^{1}, \omega_{i k}^{0}$ are the synaptic excitation and inhibition weights, respectively.

It is worth mentioning that, on this concept of distributed computing over the communication channel, can be the key that explains the efficiency of the nervous system, due to this model underlines the possibility that the fundamental processing of information is not only executed at the cellular bodies.

In summary, the morphological neural computing model with dendrite processing has the following characteristics: We have several input neurons, one of output, the output neuron can have several dendrites, each of the input neurons can excite or inhibit the corresponding axonal branches, thus the result, $y$, of the output neuron is computed as (see Figure 2):

$$
y=f\left(\bigwedge_{k=1}^{K} D_{k}(\mathbf{x})\right)
$$

where $D_{k}(\mathrm{x})$ is the output of the $k$-th dendrite when pattern $\mathbf{x}$ is input. Each $D_{k}(\mathrm{x})$ is obtained as follows

$$
D_{k}(\mathbf{x})=P_{k} \Lambda_{i \in I} \Lambda_{l \in L}(-1)^{(1-l)}\left(x_{i}+\omega_{i k}^{l}\right), \quad \mathbf{x}=\left(x_{1}, \ldots, x_{n}\right) \in R^{n}
$$

Factor $P_{k} \in\{1,-1\}$. Classification function $f(\mathbf{x})=1$ if and only if its argument is greater o equal to one (on-region) and zero otherwise (off-region). Therefore, the above-mentioned structure provides a solution to a binary classification problem.

It is worth mentioning that the inhibition signal always carries a negative sign independently of the sign of its corresponding synaptic weight.
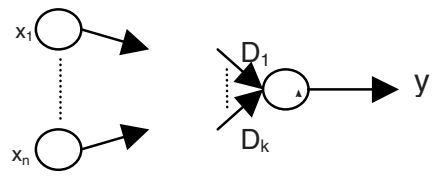

Fig. 2. Morphological neural computing model. 


\subsection{Geometric Approach of Dendrite Morphological Computation}

In [4], it is enunciated and demonstrated a Theorem that is the base of the neural morphological model with dendrite computation. In a few words this result tells us that if $X \in R^{n}$ is a compact set of patterns, then there exists a morphological neural net that classifies $X$ as its on-region and its complements as its off-region.

The above-mentioned Theorem comes together with an iterative algorithm that allows determining; given a set $X$, the parameters of the morphological neural net. For the details refer to [4].

However, the geometrical counterpart of all the analytic and algorithmic statements does not appears completely clear. Since a didactical point of view and of the correct assimilation of the concepts it is desirable to develop until possible an intuitive and geometrical idea of the formal aspects. It is worth mentioning that the proposed intuitive geometrical vision is not only important for a better understanding of the concepts, it will also allow to efficiently to develop a construction algorithm as occurs with the analytical tools as we will next see.

a. To begin characterizing the geometrical approach, let us consider the effect of the axonal branches over the dendrites:

$$
\bigwedge_{l \in L}(-1)^{1-l}\left(x_{i}+\omega_{i k}^{l}\right)=\Lambda\left\{\left(x_{i}+\omega_{i k}^{1}\right),-\left(x_{i}+\omega_{i k}^{0}\right)\right\}
$$

This expression is the intersection between two lines that cut axe $x_{i}$ at $-\omega_{i k}^{1}$ and $-\omega_{i k}^{0}$, respectively (see Figure 3).

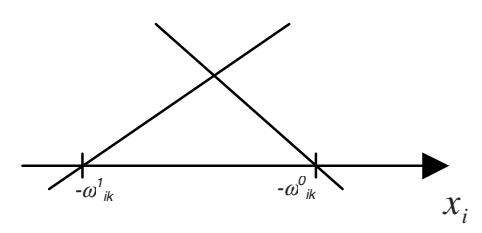

Fig. 3. Incidence of axonal branches over a dendrite.

In this case the firing region consists on the base of the triangle that is formed when the lines intersect, the complement constitutes the off-region (in the firing regions operator $\Lambda$ takes positive or zero values; in the offregion it takes negative values).

b. At each dendrite fall axonal branches from several input neurons; each of them define an on or off-region; all of them interact according to the following expression

$$
D \tau_{k}\left(\mathrm{x}^{\xi}\right)=P_{k} \Lambda_{i \in I} \Lambda_{l \in L}(-1)^{(l \in L)}\left(x_{i}^{\xi}+\omega_{i k}^{l}\right)
$$

where $x^{\xi}=\left(x_{1}^{\xi}, \ldots, x_{n}^{\xi}\right) \in R^{n}$ is an input pattern, $\xi=1,2, \ldots, m$. 
Due to the intersection is given by operator $\Lambda$ and each pair of axonal branches corresponds to different axes $x_{i}(i=1, \ldots, n)$, this makes that the firing conjoint regions are formed with the Cartesian products of the corresponding firing regions at each input (see Figure 4).

When $P_{k}=-1$, the firing region becomes an off-region and vice versa. It is important to note that the firing border frontier remains on, even when $P_{k}=-1$.

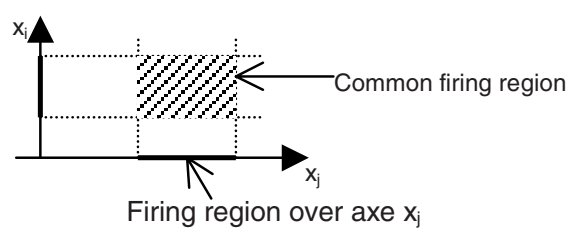

Fig. 4. Conjoint firing region.

Finally, the computation of the output is done as:

$$
y(\mathbf{x})=f\left(\bigwedge_{k=1}^{K} D_{k}(\mathbf{x})\right)
$$

This implies that the firing region of the net is the intersection of the regions of each dendrite (Figure 5).

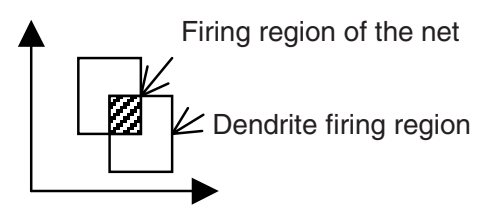

Fig. 5. Firing region of the morphological net.

In short, the firing region of all the net, what is what we want to characterize, is obtained by forming the firing regions (one for each input variable), by applying Cartesians products between firing regions or their complements, to finally get in general a hyper-rectangle as the firing region and its complement as the off-region. All the involved parameters in the above process can be synthetisized in a table as the one shown in Figure 6.

At each row appear the synaptic weights of the axonal branches that fall on the corresponding dendrite, on last column $P_{k}$ specifies if the firing region is complemented or not. 


\begin{tabular}{|c|c|c|c|c|}
\hline$D_{k}$ & $\omega_{i k}^{1}$ & $\omega_{i k}^{0}$ & $\cdots$ & $P_{k}$ \\
\hline $\mathrm{D}_{1}$ & & & & \\
\hline$\vdots$ & & & & $\vdots$ \\
\hline $\mathrm{D}_{K}$ & & & & \\
\hline
\end{tabular}

Fig. 6. Parameters of the morphological net.

\section{Experimental Results}

The algorithm in geometrical terms is composed of two steps:

1. Find the hyper-rectangle covering all patterns belonging to class $C_{1}$ (firing patterns), although patterns of class $C_{0}$ (turn-off patterns) are included.

2. Isolate the points belonging to class $C_{0}$ in maximal neighborhoods and take the complement of these neighborhoods so that the neighborhoods become part of the off-region.

Example 4.1. Let $X=[(1,4),(2,5),(2,2),(3,2),(3,3),(4,4),(5,1)]$ patterns of class $C_{l}(\bullet)$ and $X^{1}=[(2,3),(3,4),(4,3)]$ of class $C_{0}(0)$ :

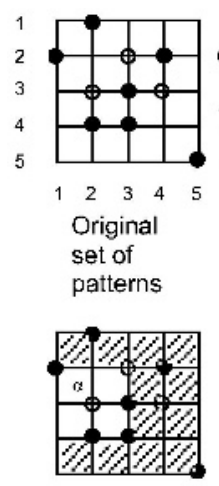

Maximal isolating neighborhood of pattern $\alpha$ class $\mathrm{C}_{0}$ that is inside the firing region
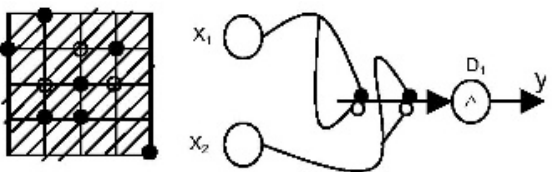

\begin{tabular}{|l|l|l|l|l|l|}
\hline$D_{k}$ & $\omega_{1 k}{ }^{j}$ & $\omega_{3 k}{ }^{0}$ & $\omega_{2 k}{ }^{j}$ & $\omega_{2 k}{ }^{0}$ & $P_{k}$ \\
\hline $\mathrm{D}_{1}$ & -1 & -5 & -1 & -5 & 1 \\
\hline
\end{tabular}

Firing region of the rectangle covering the patterns of $\mathrm{C}_{1}$
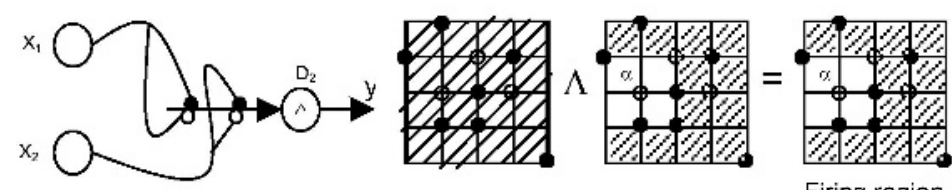

Firing region taking into account $\mathrm{D}_{1}$ and $\mathrm{D}$.

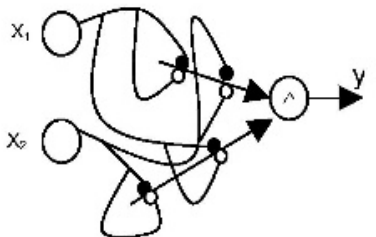

\begin{tabular}{|c|c|c|c|c|c|}
\hline$\overline{D_{t}}$ & $\sigma_{i,}{ }^{i}$ & $\overline{\omega_{i}{ }^{b}}$ & $\omega_{n k}{ }^{\prime}$ & $\left(^{(1)}{ }^{\circ}{ }^{0}\right.$ & $P_{k}$ \\
\hline D. & -1 & -5 & -1 & -5 & 1 \\
\hline $\mathrm{D}_{2}$ & -1 & -3 & -2 & -4 & -1 \\
\hline
\end{tabular}



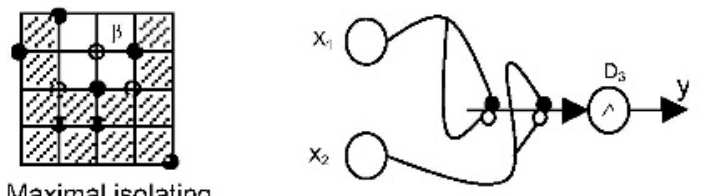

\begin{tabular}{|l|l|l|l|l|l|}
\hline$D_{k}$ & $\omega_{i k}{ }^{\prime}$ & $\omega_{3 i}{ }^{n}$ & $\omega_{2 k}{ }^{\prime}$ & $\omega_{22}{ }^{\prime}$ & $P_{k}$ \\
\hline $\mathrm{D}_{3}$ & -2 & -4 & -3 & -5 & -1 \\
\hline
\end{tabular}

Maximal isolating

neighborhood of pattern $\beta$

class $\mathrm{C}_{0}$ that is inside the

firing region

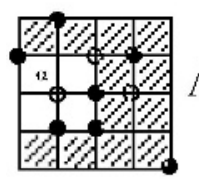

$\Lambda$

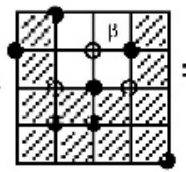

Firing region

taking into

account $\mathrm{D}_{1}, \mathrm{D}_{2}$

and $\mathrm{D}_{3}$

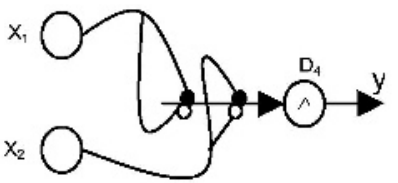

\begin{tabular}{|l|l|l|l|l|l|}
\hline$D_{k}$ & $\omega_{2 \hbar}{ }^{2}$ & $\omega_{2 k}{ }^{0}$ & $\omega_{2 k}{ }^{i}$ & $\omega_{2 k}$ & $l_{k}{ }_{k}$ \\
\hline $\mathrm{D}_{4}$ & -3 & -5 & -2 & -4 & -1 \\
\hline
\end{tabular}

\begin{tabular}{|l|l|l|l|l|l|}
\hline$D_{k}$ & $\omega_{h k}{ }^{2}$ & $\omega_{k}{ }^{2}$ & $\omega_{2 k}{ }^{i}$ & $\omega_{2 k}{ }^{g}$ & $P_{k}$ \\
\hline $\mathrm{D}_{1}$ & -1 & -5 & -1 & -5 & 1 \\
\hline $\mathrm{D}_{2}$ & -1 & -3 & -2 & -4 & -1 \\
\hline $\mathrm{D}_{3}$ & -2 & -4 & -3 & -5 & -1 \\
\hline
\end{tabular}

Maximal isolating neighborhood of pattern $\chi$ class $\mathrm{C}_{0}$ that is inside the firing region
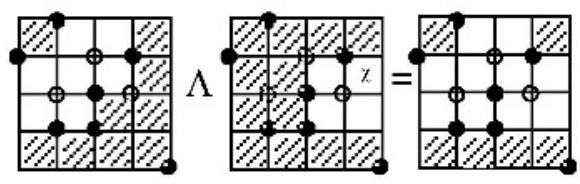

\begin{tabular}{|c|c|c|c|c|c|}
\hline$D_{k}$ & $\omega_{i k}{ }^{I}$ & $\omega_{3 \pi}{ }^{\prime \prime}$ & $\omega_{3 n^{3}}$ & $\omega_{2 n^{n}}$ & $I_{k}$ \\
\hline$D_{1}$ & -1 & -5 & -1 & -5 & 1 \\
\hline $\mathrm{D}_{2}$ & -1 & -3 & -2 & -4 & -1 \\
\hline $\mathrm{D}_{3}$ & -2 & -4 & -3 & -5 & -1 \\
\hline$D_{4}$ & -3 & -5 & -2 & -4 & -1 \\
\hline
\end{tabular}

Firing region taking into account $\mathrm{D}_{1}, \mathrm{D}_{2}, \mathrm{D}_{3}$ and $\mathrm{D}_{4}$.

To verify that the net has been effectively derived, let us classify patterns: $(1,4)$ and $(2,3)$ belonging to classes $C_{1}$, and $C_{0}$, respectively.

With $(1,4)$, we have:

$$
\begin{aligned}
& f\left(\left[\begin{array}{l}
1 \\
4
\end{array}\right]\right)=f\left(\bigwedge_{k=1}^{4} D_{k}\left[\begin{array}{l}
1 \\
4
\end{array}\right]\right) \\
& D_{1}\left(\left[\begin{array}{l}
1 \\
4
\end{array}\right]\right)=\bigwedge_{i=1}^{2} \bigwedge_{l=0}^{1}(-1)^{1-l}\left(x_{i}+\omega_{i 1}^{l}\right) \\
& =\wedge\left\{\wedge\left[\left(x_{1}+\omega_{11}^{1}\right),-\left(x_{1}+\omega_{11}^{0}\right)\right] \wedge\left[\left(x_{2}+\omega_{21}^{1}\right),-\left(x_{2}+\omega_{21}^{0}\right)\right]\right\} \\
& =\wedge\{\wedge[(1+-1),-(1-5)] \wedge[(4-1),-(4-5)]\} \\
& =\wedge\{\wedge[0,4] \wedge[3,1]\}=\wedge\{0,1\}=0
\end{aligned}
$$


In the same way, $D_{2}\left(\left[\begin{array}{l}1 \\ 4\end{array}\right]\right)=0, D_{3}\left(\left[\begin{array}{l}1 \\ 4\end{array}\right]\right)=1$ and $D_{4}\left(\left[\begin{array}{l}1 \\ 4\end{array}\right]\right)=2$.

Thus $f\left(\left[\begin{array}{l}1 \\ 4\end{array}\right]\right)=f\left(\bigwedge_{k=1}^{4} D_{k}\left[\begin{array}{l}1 \\ 4\end{array}\right]\right)=f(\wedge\{0,0,1,2\})=f(0)=1$, and $\left[\begin{array}{l}1 \\ 4\end{array}\right] \in C_{1}$.

With $(2,3)$, we have:

$$
f\left(\left[\begin{array}{l}
2 \\
3
\end{array}\right]\right)=f\left(\bigwedge_{k=1}^{4} D_{k}\left[\begin{array}{l}
2 \\
3
\end{array}\right]\right)
$$

Also $D_{1}\left(\left[\begin{array}{l}2 \\ 3\end{array}\right]\right)=1, D_{2}\left(\left[\begin{array}{l}2 \\ 3\end{array}\right]\right)=-1, D_{3}\left(\left[\begin{array}{l}2 \\ 3\end{array}\right]\right)=0$ and $D_{4}\left(\left[\begin{array}{l}2 \\ 3\end{array}\right]\right)=1$.

Thus $f\left(\left[\begin{array}{l}2 \\ 3\end{array}\right]\right)=f\left(\wedge_{k=1}^{4} D_{k}\left[\begin{array}{l}2 \\ 3\end{array}\right]\right)=f(\wedge\{1,-1,0,1\})=f(-1)=0$, and $\left[\begin{array}{l}2 \\ 3\end{array}\right] \in C_{0}$.

\section{Conclusions and Future Work}

It has been shown that the morphological neural model with dendrite computation is more intuitive from a geometrical point of view, this has been manifested by the given example, where the final table can be also obtained by following the algorithm proposed by Ritter in [4].

As future work we are working on a visual computational tool that automatically allows determining the final parameters of a net as we did in the example; and this with the aim to account with didactic tool to facilitate the training of the morphological neural model with dendrite computation.

Acknowledgments. This work was economically supported by the CIC-IPN, the CONACYT under grant 41529, the CGPI-IPN under grant 20020214 and the COFAA-IPN.

\section{References}

1. Donna L. Hudson, Neural Networks and Artificial Intelligence for Biomedical Engineering, IEEE Press, 2000.

2. Gerard X. Ritter, Joseph N. Wilson, Computer Vision Algorithms in Image Algebra, CRC, 2001.

3. Jorge Martínez, Ordered Algebraic Structures, Kluwer Academic, 2002.

4. Gerard X. Ritter, Gonzalo Urcid, Lattice Algebra Approach to Single Neuron Computation, IEEE Transactions on Neural Networks, May 2003. 